\title{
DETERMINAÇÃO DE RESÍDUOS DE AVERMECTINAS EM FÍGADO BOVINO POR CROMATOGRAFIA LÍQUIDA DE ALTA EFICIÊNCIA ${ }^{1}$
}

\author{
Scheilla V. C. SOUZA ${ }^{2, *}$, Gilsara SILVA ${ }^{3}$, Maria Helena G. M. DINIZ ${ }^{3}$, \\ Eleonora V. SANTOS ${ }^{3}$, Josefa A. LIMA ${ }^{3}$, João C. TEODORO ${ }^{3}$
}

\section{RESUMO}

Foi padronizado e validado um método analítico para determinação de resíduos de abamectin, doramectin e ivermectin em figado bovino empregando extração com acetonitrila, purificação com cartucho de $\mathrm{C}_{18}$, derivatização, detecção e quantificação por CLAE/FL. Nos ensaios com amostras adicionadas de solução padrão em niveis entre 5 e $100 \mu \mathrm{g} / \mathrm{kg}$ as recuperações médias obtidas variaram de 69,0 a $101,4 \%$ com valores de coeficiente de variação entre 5,5 e $28,1 \%$. O limite de detecção e quantificação do método, para os três residuos estudados, foi $5 \mu \mathrm{g} / \mathrm{kg}$.

Palavras-chave: abamectin, doramectin, ivermectin, resíduos, figado, cromatografia líquida de alta eficiência.

\section{SUMMARY}

DETERMINATION OF AVERMECTINS RESIDUES IN BOVINE LIVER BY HIGH PERFORMANCE LIQUID CHROMATOGRAPHY. A method for determination of abamectin, doramectin and ivermectin residues in bovine liver was standardized and validated using acetonitrile extraction, clean-up with nd $\mathrm{C}_{18}$ cartridges, derivatization, detection and quantification by HPLC/FL. In the assays using spiked samples in levels varying from 5 to $100 \mu \mathrm{g} / \mathrm{kg}$ the average recovery values ranged from 69.0 to $101.4 \%$ with coefficient of variation values between 5.5 and $28.1 \%$. The limit of detection and quantification of this method, for each studied residue, was $5 \mu \mathrm{g} / \mathrm{kg}$.

Keywords: abamectin, doramectin, ivermectin, residues, liver, high performance liquid chromatography.

\section{1 - INTRODUÇÃO}

Abamectin, doramectin e ivermectin são compostos pertencentes ao grupo das avermectinas, lactonas macrocíclicas, quimicamente relacionadas e naturalmente produzidas a partir do Streptomyces avermectilis [2]. Possuem alta atividade antiparasitária, aliada à dosagens únicas e extremamente baixas [4], administradas via oral ou injetável $[5,20]$. Causam a paralisia de nematódeos e artrópodes susceptiveis devido à inibição do neurotransmissor ácido gama-aminobutírico [3].

As avermectinas são drogas utilizadas extensivamente em animais produtores de alimentos, sendo de grande importância a determinação do tempo de retirada destes medicamentos [17] e o monitoramento de seus resíduos em tecidos animais destinados à alimentação humana [18], devido ao risco de efeitos no sistema nervoso central $[3,4]$. Os tecidos alvos sugeridos para monitoramentos são figado, órgão de metabolização destas drogas, e tecido adiposo, devido ao caráter lipofilico das avermectinas $[6,11,15]$.

A União Européia determinou limites máximos de $20 \mu \mathrm{g} / \mathrm{kg}$ para abamectin e $100 \mu \mathrm{g} / \mathrm{kg}$ para doramectin e ivermectin em figado bovino, e de $10 \mu \mathrm{g} / \mathrm{kg}$ para abamectin, $150 \mu \mathrm{g} / \mathrm{kg}$ para doramectin e $40 \mu \mathrm{g} / \mathrm{kg}$ para ivermectin em tecido adiposo bovino, sendo proibida a

1. Recebido para publicação em 20/04/2002. Aceito para publicação em 23/07/2002.

2. Unidade de Garantia da Qualidade, Laboratório Regional de Apoio Animal (LARA/MG), Ministério da Agricultura, Pecuária e Abastecimento (MAPA), Tel. (31) 36613000, Fax (31) 36612383, e-mail labmg@yahoo.com.br

3. Setor de Cromatografia, Laboratório Regional de Apoio Animal (LARA) MG), Ministério da Agricultura, Pecuária e Abastecimento (MAPA), Tel. (31) 36613000, Fax (31) 36612383, e-mail labmg@yahoo.com.br * A quem a correspondência deve ser enviada. utilização de doramectin em bovinos produtores de leite para consumo humano [11]. O CODEX ALIMENTARIUS estipulou um limite máximo de resíduo de $100 \mu \mathrm{g} / \mathrm{kg}$ para cada um dos três resíduos em figado bovino e de $100 \mu \mathrm{g} / \mathrm{kg}$ para abamectin, $150 \mu \mathrm{g} / \mathrm{kg}$ para doramectin e $40 \mu \mathrm{g} / \mathrm{kg}$ para ivermectin em tecido adiposo bovino [7]. Enquanto no Brasil foi estabelecido um limite máximo de resíduo de $100 \mu \mathrm{g} / \mathrm{kg}$ para ivermectin em figado bovino [1].

A confiabilidade dos estudos relativos à presença destes compostos em alimentos de origem animal e outras matrizes é extremamente dependente da eficiência do método analítico empregado [22]. Os primeiros métodos publicados para determinação de avermectinas empregavam purificação por extração em fase sólida e partição líquido:líquido seguidas de determinação por cromatografia líquida de alta eficiência (CLAE), em fase normal, com detecção por ultra-violeta (UV) [16, 21]. Posteriormente, métodos mais rápidos foram desenvolvidos empregando purificação com cartuchos de extração em fase sólida, seguidos de determinação por CLAE/UV em fase reversa [15]. Métodos empregando purificação, seguida de derivatização para um composto fluorescente e posterior determinação por CLAE, detecção de fluorescência (FL) têm sido amplamente utilizados $[9,12,13,17,19$, $22,23]$ e recomendados por organismos oficiais de regulamentação $[1,7]$.

O presente trabalho objetivou otimizar e validar um método multiresíduo para determinação das avermectinas abamectin, doramectin e ivermectin em figado bovino, baseado nos descritos por CRAYG [9] e ROUDAUT [19], para atender as atividades de monitoramento do Laboratório Regional de Apoio Animal (LARA/MG), integrantes do Plano Nacional de Controle de Resíduos Biológicos em Produtos de Origem Animal do Ministério da Agricultura, Pecuária e Abastecimento (MAPA). 


\section{2 - MATERIAL E MÉTODOS}

\section{1 - Amostras}

Amostras de figado bovino, livres dos resíduos pesquisados (abamectin, doramectin e ivermectin), foram utilizadas como amostras brancas, após análise segundo procedimentos do LARA/MG. Estas amostras foram identificadas, homogeneizadas, embaladas em sacos de polietileno e mantidas sob congelamento $\left(-10^{\circ} \mathrm{C}\right.$ a $\left.-17^{\circ} \mathrm{C}\right)$ até o momento das análises.

\section{2 - Padrões}

As soluções dos padrões de abamectin, e ivermectin, ambos da marca Merck Sharp \& Dohme (Rahway, NJ) e de doramectin, da marca Pfizer (Nagoya, Japan), foram preparadas em metanol, identificadas, acondicionadas, avaliadas (em termos de intensidade do sinal e tempo de retenção das substâncias pesquisadas) e armazenadas, sob congelamento $\left(-10^{\circ} \mathrm{C}\right.$ a $\left.-17^{\circ} \mathrm{C}\right)$, até o momento das análises. As soluções estoque foram preparadas com $10 \mu \mathrm{g} / \mathrm{mL}$ de cada substância enquanto a solução para adição às amostras (pool) foi preparada com 500ng/mL de cada substância.

\section{3 - Procedimentos analiticos}

Os procedimentos analiticos padronizados e validados foram baseados nos descritos por CRAYG [9] e ROUDAUT [19].

\subsection{1 - Extração}

Após o descongelamento da amostra de figado bovino, até temperatura suficiente para o corte, foram pesados $5 \mathrm{~g}$, acrescentados $15 \mathrm{~mL}$ de acetonitrila e extraídos os resíduos de abamectin, doramectin e ivermectin por centrifugação durante 10 minutos a $3500 \mathrm{rpm}$, a $25^{\circ} \mathrm{C} \pm$ $5^{\circ} \mathrm{C}$.

\subsection{2 - Purificação}

Os extratos, adicionados a $40 \mathrm{~mL}$ de água e $50 \mu \mathrm{L}$ de trietilamina, foram percolados em cartuchos Waters de $\mathrm{C}_{18} 6 \mathrm{~mL} / 500 \mathrm{mg}$ (previamente condicionados com $5 \mathrm{~mL}$ de acetonitrila e $5 \mathrm{~mL}$ de acetonitrila:água 30:70, v/v, contendo $0,1 \%$ de trietilamina) e posteriormente eluídos com $5 \mathrm{~mL}$ de acetonitrila.

\subsection{3 - Derivatização}

Após evaporação do solvente $\left(45^{\circ} \mathrm{C}\right)$ sob fluxo de nitrogênio, a derivatização dos extratos purificados das amostras e das soluções padrões foi realizada por adição de $150 \mu \mathrm{L}$ da mistura derivatizante $(200 \mu 1$ de 1-metilimidazol, $600 \mu \mathrm{L}$ de anidrido acético e $900 \mu \mathrm{L}$ de dimetilformamida), seguida de aquecimento por $60 \mathrm{mi}-$ nutos, entre $95^{\circ} \mathrm{C}$ e $100^{\circ} \mathrm{C}$, em bloco aquecedor. Após o resfriamento à temperatura ambiente, a derivatização completa foi indicada pela formação de um extrato marrom escuro.

\subsection{4 - Separação, detecção e quantificação}

Os extratos derivatizados foram posteriormente completados para $1 \mathrm{~mL}$ com metanol, filtrados em membrana Millipore de $0,45 \mu \mathrm{m}$ e analisados por CLAE (sistema de bomba quaternário, degaseificador de membrana, injetor automático e detector FL Termo Separation Products; aquisição, controle e processamento de dados por microcomputador IBM), sob as seguintes condições:

- detector de fluorescência com comprimentos de onda de excitação e emissão a 364 e 426 nm, respectivamente;

- coluna Lichrosorb RP 18, $7 \mu \mathrm{m}, 250 \mathrm{~mm}$ x $4 \mathrm{~mm}$ e précoluna Lichrospher $100 \mathrm{RP} 18,4 \mathrm{~mm} \times 4 \mathrm{~mm}$;

- fase móvel metanol;

- fluxo de 0,9mL/min;

- tempo de separação de 12 minutos para os três resíduos;

- volume de injeção loop de $20 \mu \mathrm{L}$;

- integração pelo parâmetro altura.

\section{4 - Procedimentos de validação}

A validação do método foi conduzida segundo o procedimento de validação intralaboratorial do Setor de Cromatografia do LARA/MG [8, 10, 14].

\subsection{1 - Ensaios com padrões}

Nos ensaios com soluções padrões (pools) foram avaliados a linearidade sem interferência da matriz e o limite de detecção do equipamento $[8,10,14]$. As soluções utilizadas foram de $0 ; 12,5 ; 25 ; 125 ; 250$ e $500 \mathrm{ng} / \mathrm{mL}$.

\subsection{2 - Ensaios com amostras adicionadas de so- lução padrão das substâncias pesquisadas}

As amostras (2.1) foram adicionadas de solução padrão, nos níveis de 5; 7; 10; 25; 50 e $100 \mu \mathrm{g} / \mathrm{kg}$, em pelo menos cinco repetições. A cada grupo de amostras foi incluída uma amostra em branco. Também foram realizados ensaios com sete amostras adicionadas de solução padrão, em níveis desconhecidos pelo analista, entre 25 e $100 \mu \mathrm{g} / \mathrm{kg}$.

Nestes ensaios foram avaliados especificidade, exatidão, precisão, limites de detecção e quantificação do método $[8,10,14]$.

\subsection{3 - Valores de referência}

Foram adotados como referência para avaliação da exatidão e precisão os valores estabelecidos como aceitáveis pelo CODEX [8] e internalizadas pelo MERCOSUL [14] para métodos quantitativos (Tabela 1).

\section{3 - RESULTADOS E DISCUSSÃO}

Nos ensaios com soluções padrões $(0 ; 12,5 ; 25 ; 125$; 250 e $500 \mathrm{ng} / \mathrm{mL}$ ) somente não foram detectados 
abamectin, doramectin e ivermectin na solução de $12,5 \mathrm{ng} / \mathrm{mL}$. Para as demais soluções picos dos três compostos foram registrados, com sinais três vezes maior que o valor do maior sinal de ruído nos cromatogramas. Desta forma, foi demonstrada linearidade da resposta, com coeficientes de determinação superiores a 0,995, na faixa de 25 a $500 \mathrm{ng} / \mathrm{mL}$.

TABELA 1. Niveis de concentração e respectivos valores de recuperação e coeficiente de variação aceitáveis para resultados quantitativos

\begin{tabular}{ccc}
\hline $\begin{array}{c}\text { Concentração } \\
(\mu \mathrm{g} / \mathrm{kg} \text { ou } \mathrm{L})\end{array}$ & $\begin{array}{c}\text { Exatidão } \\
\text { Recuperação }\end{array}$ & $\begin{array}{c}\text { Precisão } \\
\text { Coeficiente de variação } \\
\text { intralaboratorial }(\%)\end{array}$ \\
\hline$\leq 1$ & 50 a 120 & 35 \\
1 a 10 & 60 a 120 & 30 \\
10 a 100 & 70 a 110 & 20 \\
$>100$ & 80 a 110 & 15 \\
\hline
\end{tabular}

Fonte: CODEX [8], MERCOSUL [14].

O limite de detecção do equipamento, determinado como a menor massa da substância detectada, nas condições estabelecidas pelo método, com sinal, no minimo, três vezes o valor do maior sinal de ruído no cromatograma [8, 10, 14], foi de $0,5 \mathrm{ng}$, para cada um dos três resíduos, correspondente à massa injetada em $20 \mu \mathrm{L}$ da solução de $25 \mathrm{ng} / \mathrm{mL}$.

Especificidade foi observada nos ensaios utilizando amostras adicionadas de solução padrão. Os picos de abamectim, doramectim e ivermectim apresentaram resolução satisfatória, separados dos picos de interferentes. Houve concordância entre os tempos de retenção e

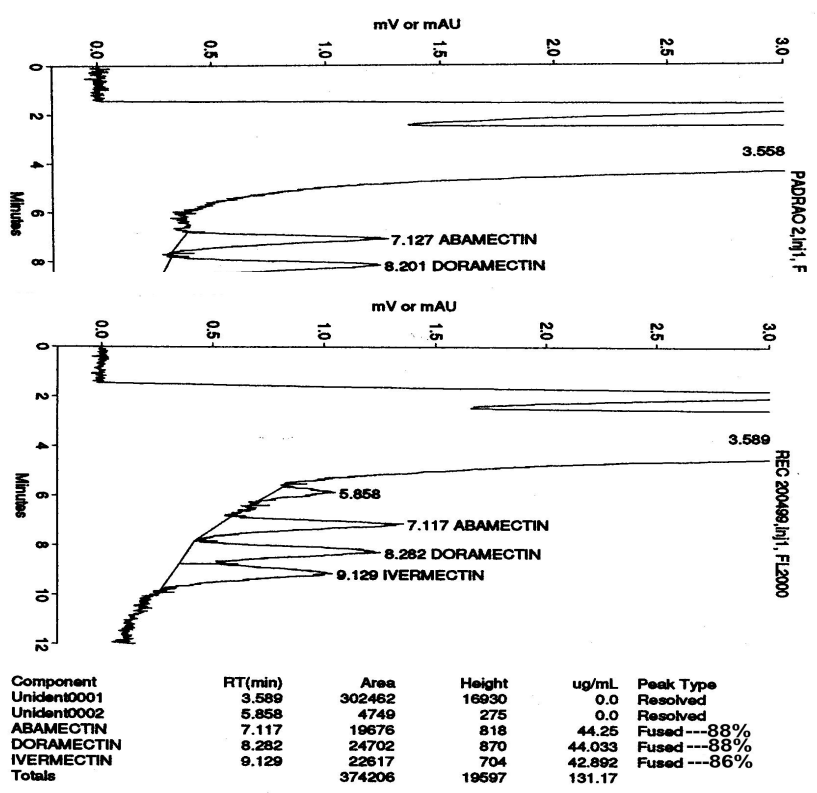

FIGURA 1. Cromatogramas obtidos para solução padrão (50ng/ $\mathrm{mL})$ e amostra adicionada de solução padrão $(50 \mathrm{mg} / \mathrm{kg})$. intensidade de resposta dos três compostos pesquisados quando comparados os cromatogramas obtidos para soluções padrões e para as amostras (Figura 1).

Os valores médios de porcentagem de recuperação variaram entre 92,9 e 101,4\% para abamectin; 91,5 e $99,5 \%$ para doramectin e 69,0 e $98,4 \%$ para ivermectin. Os coeficientes de variação apresentaram-se entre 6,1 e 23,9\% para abamectin; 5,9 e 26,8\% para doramectin e 5,5 e $28,1 \%$ para ivermectin (Tabela 2 ). Como os valores de porcentagem de recuperação médios e coeficiente de variação apresentaram-se dentro dos intervalos descritos como aceitáveis (Tabela 1) [8, 14] para todas as concentrações avaliadas, foi determinado como limite de detecção e quantificação do método o menor nível estudado $(5 \mu \mathrm{g} / \mathrm{kg})$. Avaliando-se os resultados individuais de porcentagem de recuperação (Figura 2), observa-se uma maior dispersão dos resultados, quanto menor a concentração dos analitos na matriz.
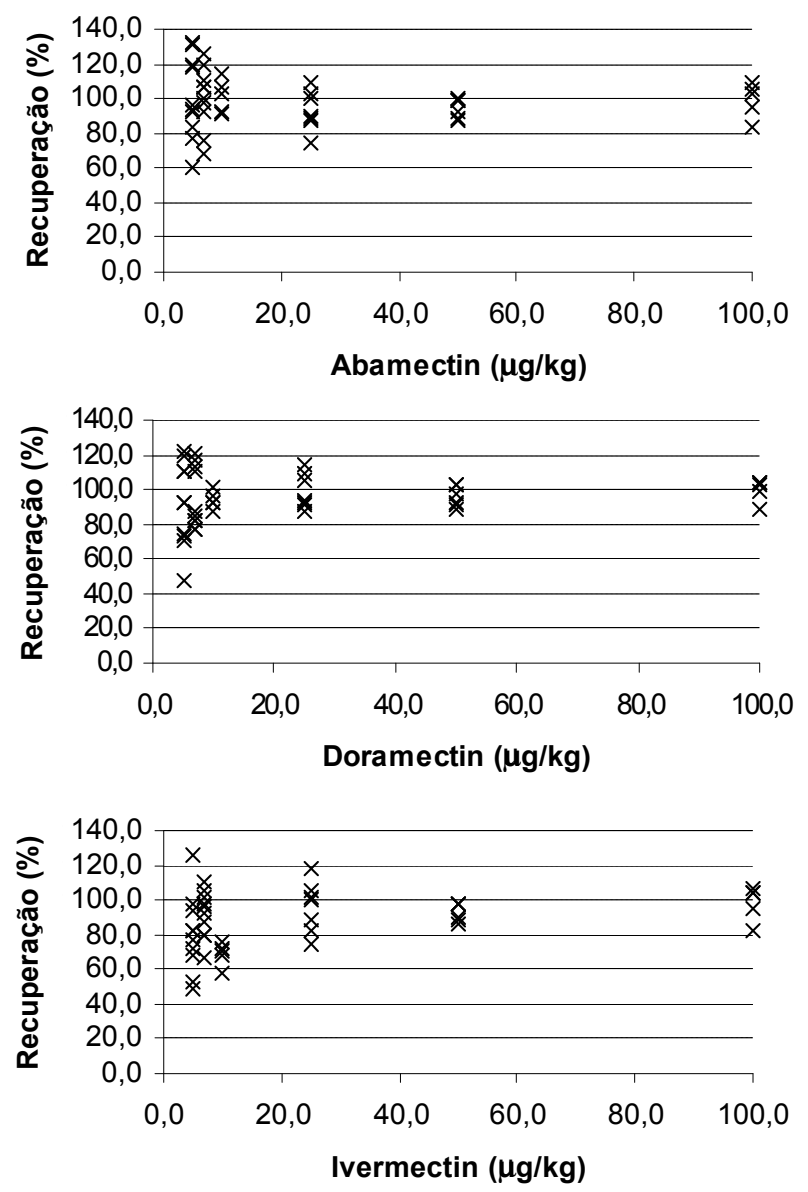

FIGURA 2. Valores individuais de porcentagem de recuperação para abamectin, doramectin e ivermectin obtidos nos ensaios com amostras adicionadas de solução padrão em 5; 7; $10 ; 25 ; 50$ e $100 \mu \mathrm{g} / \mathrm{kg}$ com $10 ; 10 ; 5 ; 7 ; 6$ e 5 replicatas, respectivamente.

Nos ensaios com amostras adicionadas de solução padrão, em concentração desconhecida pelo analista, os resultados médios de porcentagem de recuperação fo- 
ram 101,$1 ; 101,0$ e 96,7\%, com coeficientes de variação de 11,$2 ; 9,2$ e $12,5 \%$ para abamectin, doramectin e ivermectin, respectivamente. Na Figura 3 pode ser observada a dispersão dos valores de porcentagem de recuperação obtidos para estas amostras de concentração desconhecida, os quais variaram entre 82,0 e $117,0 \%$.

TABELA 2. Valores médios de porcentagem de recuperação e coeficientes de variação obtidos para abamectin, doramectin e ivermectin por nivel de concentração estudado

\begin{tabular}{cccccccc}
\hline $\begin{array}{c}\text { Concentração } \\
(\mu \mathrm{g} / \mathrm{kg})\end{array}$ & $\mathrm{n}$ & $\mathrm{Rm}(\%)$ & C.V. $(\%)$ & $\mathrm{Rm}(\%)$ & C.V. (\%) & $\mathrm{Rm}(\%)$ & C.V. (\%) \\
\hline 100 & 5 & 99,3 & 9,9 & 99,5 & 6,7 & 98,4 & 10,2 \\
50 & 6 & 93,9 & 6,1 & 95,9 & 6,5 & 92,7 & 5,5 \\
25 & 7 & 92,9 & 12,6 & 99,1 & 10,6 & 95,9 & 15,3 \\
10 & 5 & 101,4 & 9,4 & 94,0 & 5,9 & 69,0 & 9,8 \\
7 & 10 & 100,4 & 17,9 & 95,2 & 18,7 & 91,5 & 14,5 \\
5 & 10 & 100,5 & 23,9 & 91,5 & 26,8 & 80,1 & 28,1 \\
\hline
\end{tabular}

$\mathrm{Rm}$ = recuperação média (a média dos valores individuais de porcentagem de recuperação calculados pela variação entre o valor do resultado obtido no ensaio e o valor real do analito adicionado à matriz, expresso em porcentagem do valor real). C.V. = coeficiente de variação. $\mathrm{n}=$ número de replicatas.

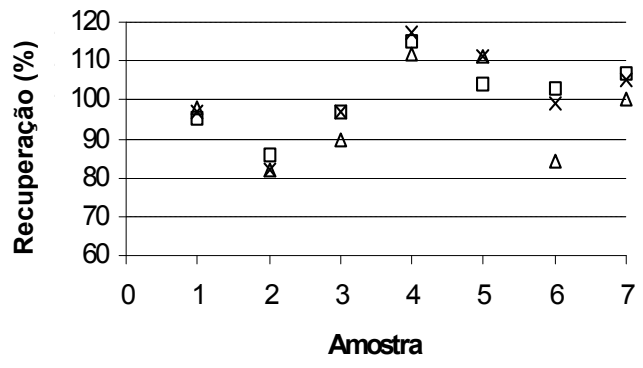

$\times$ Abamectin $\quad$ DDoramectin $\quad \Delta$ lvermectin

FIGURA 3. Dispersão dos valores de porcentagem de recuperação obtidos para sete amostras adicionadas de solução padrão em concentrações desconhecidas pelo analista.

Peso da amostra, volume final do extrato, temperaturas do banho de água e do bloco aquecedor, etapas de evaporação dos extratos e etapa de derivatização, foram considerados pontos críticos de controle do método em observações durante os procedimentos de validação.

\section{4 - CONCLUSÕES}

O método otimizado e validado mostrou-se eficiente em termos de linearidade, especificidade, exatidão e precisão, além de possuir limite de detecção e quantificação suficientemente baixo, para ser adotado nas atividades de monitoramento de resíduos de avermectinas em figado bovino do MAPA.

\section{5 - REFERÊNCIAS}

[1] BRASIL, Ministério da Agricultura e do Abastecimento. Instrução normativa 3. Diário Oficial da União. Brasília, 17 fev. 1999. Seção 1, p.15-33.
[2] BURG, R. W.; MILLER, B. M.; BAKER, E. E.; BIRNBAUM, J.; CURRIE, S. A.; HARTMAN, R.; KONG, Y. L.; MONAGHAN, R. L.; OSLON, G.; PUTTER, I.; TUNAC, J. B.; WALLIC, H.; STAPLEY, E. O; OIWA, R.; OMURA, S. Avermectin a new family of potent anthelmintic agents, producing organism and fermentation. Antimicrob. Agents Chemother., v. 15, p. 361, 1979.

[3] CAMPBELL, W. C.; BENZ, G. W. Ivermectin: a review of efficacy and safety. J. Vet. Pharmacology Therapy, v.7, p. 1-16, 1984.

[4] CAMPBELL, W. C.; FISHER, M. H.; STAPLEY, E. O.; ALBERSSCHONBERG, G.; JACOB, T. A. Ivermectin: a potent new antiparasitic agent. Science, v. 221, p. 77, 1983.

[5] CAMPBELL, W. C. Ivermectin and heartworm. Semin. Vet. Med. Surg., v. 2, p. 48-55, 1987.

[6] CHIU, S. H. L.; SESTOKAS, E.; TAUB, R.; GREEN, M. L.; BAYLIS, F. P.; JACOB, T. A.; LU, A. Y. H. Metabolic disposition of ivermectin in swine. J. Agric. Food Chem., v. 38, p. 2079-2085, 1990.

[7] CODEX ALIMENTARIUS. Joint FAO/WHo Expert Committee on Food Additives. Fifty-second meeting. Rome. 1999.

[8] CODEX ALIMENTARIUS. Volume 3. Residuos de medicamentos veterinarios en los alimentos. 2 ed. Roma. 1993.

[9] CRAYG, D. C. Modified method for the determination of ivermectin residues in animal tissues. J. AOAC Int., v. 76, p. 1149-1151, 1993.

[10] EURACHEM. The fitness for purpose of analytical methods. A laboratory guide to method validation and related topics. 1998. $61 \mathrm{p}$.

[11] JOCE - Jornal Oficial das Comunidades Européias. Bruxelas, 4 mar. 1999. Regulamento 508/1999. p. 27.

[12] KHUNACHAK, A.; DACUNHA, A. R.; STOUT, S. J. Liquid chromatographic detemination of moxidectin residues in cattle tissues and confirmation in cattle fat by liquid chromatography/mass spectrometry. J. AOAC Int., v. 76, p. 1230-1235, 1993.

[13] KIJAK, P. J. Liquid chromatographic determination of ivermectin in bovine milk: interlaboratory study. J. AOAC Int., v. 75, p. 747-750, 1992.

[14] MERCOSUL Mercado Comum do Cone Sul. Critérios para a validação de metodologias analiticas para determinação de residuos de principios ativos de medicamentos veterinários (RMV) em produtos de origem animal. Resolução 57/94, [s.n.1.], 1994.

[15] OEHLER, D. D.; MILLER, J.A. Liquid chromatographic determination of ivermectin in bovine serum. J. Assoc. Off. Anal. Chem., v. 72, p. 59, 1989.

[16] PIVNICHNY, J. V.; SIM, J. S. K.; ZIMMERMAN, L. A. J. Pharm. Sci., v. 72, p. 1447, 1983.

[17] PRABHU, S. V.; WEHNER, T. A.; TWAY, P. C. Determination of ivermectin levels in swine tissues at the parts per billion level by liquid chromatography with fluorescence detection. J. Agric. Food Chem., v. 39, p. 1468-1471, 1991.

[18] PRABHU, S. V.; WEHNER, T. A.; TWAY, P. C. Determination of ivermectin levels in swine tissues at the parts per billion level by liquid chromatography with fluorescence detection. J. Agric. Food Chem., v. 39, p. 1466-1471, 1991.

[19] ROUDAUT, B. Multiresidue method for the determination of avermectin and moxidectin residues in the liver using HPLC with fluorescence detection. Analyst., v. 123, p. 2541-2544, 1998. 
[20] SANGSTER, N. C. Anthelmintic resistance: past, present and future. Int. J. Parasitol., v. 29, p. 115-124, 1999.

[21] SCHNITZERLING, H. J.; NOLAR, J. Normal phase liquid chromatographic determination of nanogram quantities of ivermectin in cattle blood or plasma. J. Assoc. Off. Anal. Chem., v. 68, p.36, 1985.

[22] SKALAVOUNOS, C.; DEMERS, N.; LUKASZEWICZ, J.; NOWAKOWSKI, M. Photoisomerization of aromatic doramectin derivatives. J. Agric. Food Chem., v. 42, p. 1228-1231, 1994.

[23] USDA - UNITED STATES DEPARTMENT OF AGRICULTURE. Food Safety and Inspection Service and Technology. Analytical chemistry laboratory guidebook. Residue Chemistry. Washington: USDA. [s.n.], 1991. (paginação irregular). 